\title{
Optimized dispatch of energy storage systems in unbalanced distribution networks
}

Jeremy D. Watson, Student Member, IEEE, Neville R. Watson, Senior Member, IEEE, and Ioannis Lestas, Member, IEEE

\begin{abstract}
This paper presents a method to achieve optimal active and reactive power contributions from each energy storage system in an unbalanced distribution network to minimize power loss, while ensuring network current and voltage constraints are satisfied. By modelling loads as either constant current or constant impedance, the AC optimal power-flow is transformed into a noniterative convex optimization problem. The application of capacity constraints, voltage constraints, and energy storage constraints in an unbalanced three-phase four-wire system is considered, addressing specific issues pertaining to unbalanced networks such as voltage unbalance and neutral voltage displacement. The proposed method is then used to demonstrate optimized dispatch of energy storage systems in a suitable four-wire unbalanced distribution test network. The contribution of losses in the neutral wire to the total losses is also determined for a test system under a range of operating conditions and various neutral earthing systems, highlighting the importance of considering this in a typical unbalanced distribution network.
\end{abstract}

Index Terms-Energy storage systems, distribution networks, network unbalance, neutral voltage displacement, neutral earthing systems, optimal power flow, energy losses.

\section{NOMENCLATURE}

$\alpha$

$B_{i j}$ phasor rotation operator

$c_{x, \text { real }}$ susceptance between nodes $i$ and $j$

$c_{x, \text { reactive }}$

$\overline{c_{x}}$

$E_{\text {capacity, } x}$

ESS

$E S S_{\text {loss }}$

$G_{i j}$

I branch

$I_{i j}$

$I_{\text {inj }}$

l

[M]

n

$N E_{\text {loss }}$

$\eta_{x}$

$P_{\text {loss }}$

R

$R_{i j}$

$R_{N, i}$
$S$

$v$

$V_{a, m}$

$v_{i, r e a l}$

$v_{i, i m a g}$

$V n_{m}$

$V p_{m}$

$V U F_{m}$

$X_{i j}$

[Y]

$Y_{i j}$ number of controllable energy storage devices in the network vector of voltages at each node phase $a$ voltage at bus $m$; likewise phases $b, c$ real part of the complex voltage at node $i$ imaginary part of the complex voltage at node $i$ negative sequence voltage at bus $m$ positive sequence voltage at bus $m$ voltage unbalance factor at bus $m$ reactance between nodes $i$ and $j$ nodal admittance matrix of the network complex admittance between nodes $i$ and $j$

Minimum and maximum limits are denoted by underbars and overbars respectively. Vectors and matrices are in bold, while matrices [M] are also denoted by square brackets. Also for a matrix [M], we use the notation $M_{i, j}$ to denote its entry at the ith row and jth column.

\section{INTRODUCTION}

Sustainability is an increasingly important goal in the electrical power industry, and the smart grid is the key to achieving this [1]. A major aim is to increase the amount of renewable generation, especially by integrating distributed energy resources (DER) such as solar photovoltaics (PV) and wind energy. However, these sustainable energy sources are highly unpredictable, and the generation profile often does not coincide with periods of high network demand. One of the most important solutions is energy storage. Distributed energy storage systems (ESS(s)), especially battery energy storage systems (BESS(s)) have the potential to provide many benefits in many different facets [2], including primary voltage and frequency control, peak shaving and smoothing control [3], demand response, energy arbitrage, reduction of network losses, and outage avoidance. There is a consensus that BESS will play a vital role in enabling smart grids [4], however further research is needed to determine how to control and optimize BESS in the smart grid.

Energy loss is an important metric for assessing smart grid performance. The reduction of losses in the distribution system can result in significant savings [5]. Although many factors contribute to energy loss, this paper considers power loss as a fixed loss (mainly due to transformer losses [6]) plus variable losses which depend on the power allocation in the network. The variable losses are the product of the square of current and resistance in a branch, added to the loss associated with the efficiency of each ESS, and the current flowing through each neutral to ground connection multiplied by the neutral voltage. 
Given a certain total ESS injection, we aim to minimize energy losses while satisfying important network and ESS constraints. The traditional method is for the ESS contributions to be proportional to ESS capacity [7]; however, energy losses may be significantly reduced by optimally choosing this allocation. The rest of the paper is structured as follows. Section II summarizes related work; section III describes in detail the problem and formulates the corresponding optimization problem. The results of a case study are presented in section V, with conclusions drawn in section VI.

\section{RELATED WORK}

ESS dispatch problems have been considered in several contexts, e.g. [8], [9], [10]. In [9], for example, a fully distributed algorithm is proposed to find the optimal dispatch schedule for a smart grid with renewable and energy storage integration considering energy losses. However, while these studies often include the aspect of time scheduling, they tend to use either power-balance equations [8], direct current (DC) power-flow (extended to include losses in [9]), or require full non-convex alternating current (AC) load-flows [10], iterative methods [11] and/or complex nonlinear optimization methods such as particle swarm optimization [12]. The method proposed in this paper is a convex approximation of the AC optimal power-flow problem based on a nodal admittance matrix power-flow. Similar load-flow algorithms have been used in [13] [14] and [15] for unbalanced distribution networks, although these do not consider an extension to the optimal power-flow or power allocation problem. The convexity of the problem significantly improves scalability, guarantees a global optimum, and enables the implementation of distributed control schemes. It is also an important step towards the implementation of receding horizon control schemes.

Linear direct current optimal power-flow (DC OPF) approaches also have these advantages. However, DC OPF is typically unable to consider losses, reactive power-flow, or voltage constraints, although some progress in this area has been made, e.g. [16]. Linear programming (LP) models which approximate AC optimal power-flow (AC OPF) are therefore more closely related to this work. In [17] and [18], the currentvoltage (IV) formulation of the power-flow problem is explored, and this paper convexifies a similar formulation for four-wire unbalanced distribution networks. Other models such as [19] [20] [21] [22] [23] use piece-wise approximations, Taylor expansions and other similar methods to obtain a linear approximation of the AC OPF problem. However, these models often achieve a linearized load-flow via iteration, and few of these models consider unbalanced distribution systems where it is often important to consider coupling between the phases [24].

Recent innovations have seen the development of various relaxations to convexify the traditional AC optimal power-flow equations [25], and these convex relaxations have attracted considerable interest [26]. The literature includes [25], a significant contribution which shows the exact convexification of the AC OPF problem under several mild conditions for a balanced network; and [26], where relaxations used to convexify the AC OPF problem are studied. An extension to unbalanced radial networks was presented in [27]. However, these relaxations do not consider unbalance constraints and rely on certain conditions which are not always fulfilled in the distribution network. Both radial and meshed configurations exist in practice and many of these convex relaxations face difficulties when either the capacity of the branches or voltage upper limit is binding [28]. A robust quadratic AC OPF approach which considers these aspects is presented in [29] for smart distribution networks. Extensions to both meshed and unbalanced networks are also mentioned, although these aspects are not explored in detail.

The aim of the paper is to present a novel convex approximation of the optimal power-flow problem for unbalanced networks, discussing the challenges and constraints relating to network unbalance. We start from a current-injection power-flow formulation and make different assumptions to formulate the power allocation problem. The formulation has no restriction on network structure and it considers current and voltage constraints, neutral voltage displacement, and also neutral power loss [30]. Neutral currents and voltage displacements are a significant problem in practice, and energy storage systems are an important tool to mitigate this [31]. Therefore, we propose a convex optimization approach which fully integrates the neutral wire voltages and currents into the power loss minimization problem for unbalanced four-wire distribution networks, and we also consider how various parameters affect the losses in the neutral. The contributions of this study are summarized as follows:

1. The proposed formulation in the paper allows the incorporation of various important features that have not been addressed in the literature. To our knowledge, previous literature on convexified optimal power flow has not considered the case of unbalanced distribution networks in much depth, especially considering constraints regarding unbalance, power loss in the neutral wire, and meshed network configurations. These effects are important in many unbalanced distribution networks but existing methods are not able to incorporate this into a convex formulation.

2. The convex formulation proposed in the paper for optimal ESS dispatch preserves its convexity when a future horizon is considered (Appendix A). This therefore allows time scheduling to be incorporated in a computationally efficient way with models that include more advanced features as described in point 1 above.

3. This study shows that losses in the neutral should not be ignored in the OPF for distribution networks.

4. The paper demonstrates the validity of the assumptions and approximations used to convexify the optimal dispatch problem in a realistic distribution network.

5. We have shown that the reduction of energy losses achieved by an optimization approach are significant in the distribution network.

\section{DERIVATION}

\section{A. Network model}

Consider a four-wire unbalanced distribution network. The network consists of a transformer supply, four-wire conductors, 
and multiple loads and ESSs. Each node $i \in[1,2, \ldots, n]$ represents a connection on a phase or the neutral, i.e. in a fourwire system there are four nodes at each bus. The individual buses are denoted by $m \in[1,2, \ldots, \mathrm{n} / 4]$. The collection of nodes on phase $a$ are denoted by the set $A$, likewise sets $B, C$ and $N$ for phases $b, c$, and the neutral respectively. Each ESS is connected to a particular phase node and the associated neutral node, as illustrated in Fig. 1. The real and reactive contributions of the individual ESSs $x$ (the decision variables) are denoted by $c_{x, \text { real }}$ and $c_{x, \text { reactive }}$ respectively. We also use the notation $\phi(x)$ to denote the phase node ESS $x$ is connected to.

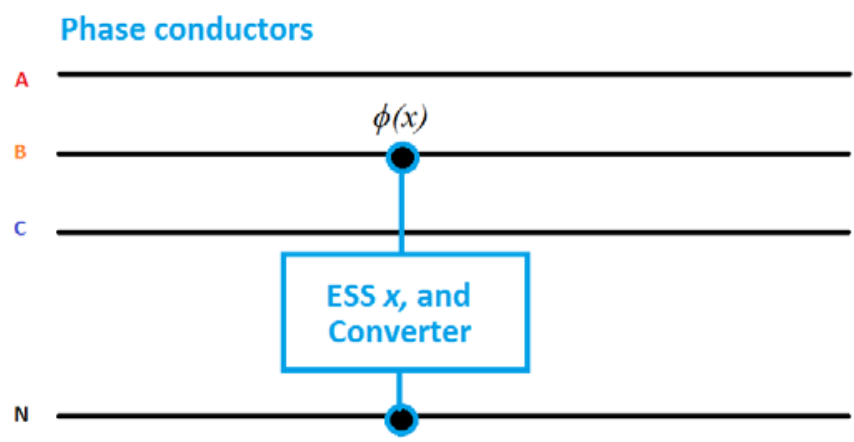

Neutral conductor

Fig. 1. Illustration of an ESS connection at phase B

The goal is to minimize energy losses in the network by optimally choosing the power allocation of each of the ESS, subject to various operational constraints. This is an optimization problem where the decision variables are the real and reactive power injections at each ESS, and the total power loss is the objective function. In this section we appropriately formulate this problem, by quantifying first the objective function and then incorporating the network constraints. Throughout this formulation various practically relevant approximations are made that render the problem convex. The significance of the problem considered is further discussed in section IV where a case study with real data is presented.

\section{B. Objective function}

The nodal admittance matrix power-flow formulation is widely used in distribution system studies [13] [15]:

$$
[Y] v=I_{i n j}
$$

where $[\boldsymbol{Y}]$ is the $n \times n$ nodal admittance matrix ( $n$ is the number of nodes in the network), $v$ is a $n \times 1$ vector containing the voltages at each node, and $\boldsymbol{I}_{\boldsymbol{i n j}}$ is a $n \times 1$ vector containing the currents drawn or injected into the network by any source or constant current load at each node. An entry of $\boldsymbol{I}_{\boldsymbol{i n j}}$ at position (node) $i, I_{i n j, i}$, will be zero if no constant current load, DER, or ESS is connected. This formulation of the nodal admittance matrix follows from Kirchhoff's Current Law and is well-studied in literature, e.g. [29] [32] including matrix representation of lines, loads, transformers, and generators.

Assumption 1: The loads may be modelled as either constant current or constant impedance.

Remark 1: Constant current loads are often used in distribution network studies [13]. In [33], constant current loads were found to be the preferred model for examining losses during the winter months. Although relatively few loads are constant current, a mixture of constant power and constant impedance loads often behaves like a constant current load. The equation for a constant power load is:

$$
I=\frac{P-j Q}{V^{*}}
$$

where $I$ is the current drawn by the load, $\mathrm{V}$ is the voltage at the node, and $P$ and $Q$ are the real and reactive load powers. A constant impedance load is governed by:

$$
I=\frac{V}{Z}, P+j Q=\frac{V V^{*}}{Z^{*}}
$$

where $Z$ is the constant impedance of the load. It is evident that the current drawn by a constant power load is inversely proportional to the voltage magnitude while the current drawn by a constant impedance load is proportional to the voltage magnitude. Hence, constant current loads are a reasonable approximation of a mixture of constant power and constant impedance loads.

Assumption 2: The ESS contributions may also be modelled as current injections. More precisely,

$$
I_{i n j, \varphi(x), E S S} \cong \frac{c_{x, \text { real }}-j c_{x, \text { reactive }}}{\operatorname{conj}\left(V_{\varphi}\right)} \text { for all ESSs } x
$$

where $I_{i n j, \varphi(x), E S S}$ is the contribution from ESS $x$ to the $I_{i n j, \varphi}$ term, and $V_{\varphi}$ is the constant nominal, estimated or measured voltage at node $\phi$, the ESS connection.

Remark 2: Note that equation (2) allows us to relate the decision variables (the real and reactive contribution at each ESS: $c_{x, \text { real }}, c_{x, \text { reactive }}$ for all ESSs $x$ ) with the entries of $\boldsymbol{I}_{\boldsymbol{i n j}}$. This is a reasonable approximation as the voltage deviations are small in magnitude compared to the voltage $V_{\varphi}$. The nominal network voltage is used unless measured voltage data or other voltage estimates are known. The accuracy of the approximations in Assumptions 1 and 2 may often be improved using estimated voltages from a previous solution or a preliminary solution of the network (1) instead of the nominal network voltage. Since these voltages are used as constants, the convexity of the problem is preserved.

In the four-wire system, single-phase loads are represented as either constant impedances between the phase wire and the neutral wire, or as a constant current drawn at the phase wire and a constant current injection of equivalent magnitude and angle into the neutral wire in the $\boldsymbol{I}_{\boldsymbol{i n j}}$ term. ESSs are modelled by a constant current injection at either the phase wire or the neutral wire, and the current injection with opposite sign at the other wire. Similarly, for ESSs integrated with DER, DER output powers can be modelled in the same $\boldsymbol{I}_{\boldsymbol{i n j}}$ term without requiring further modifications to the optimization problem. The DER will charge the ESS while the ESS contribution to the grid is determined by optimization.

The neutral wire is explicitly represented in this model if losses or voltages in the neutral wire are to be considered, otherwise Kron's reduction may be used (as in most power-flow software [34]). Section V will investigate the importance of 
modelling the neutral wire explicitly by determining how losses in the neutral are affected by several parameters.

The $Y$ matrix in equation (1) is invertible. Hence:

$$
v=[Y]^{-1} I_{i n j}
$$

The $[\boldsymbol{Y}]^{\mathbf{- 1}}$ matrix is sometimes also known as the $\mathrm{Z}$ bus matrix in literature [14]. Equation (3) thus expresses each voltage as a linear combination of the injected currents. Note that some of the terms in $\boldsymbol{I}_{\boldsymbol{i n j}}$ represent the ESS contributions which will be the decision variables for the optimization problem considered. Equation (3) is complex as both $[\boldsymbol{Y}]^{-\mathbf{1}}$ and $\boldsymbol{I}_{\boldsymbol{i n j}}$ are likely to contain complex terms; however, by splitting $\boldsymbol{I}_{\boldsymbol{i n j}}$ into real and imaginary parts ( $\boldsymbol{I}_{\boldsymbol{i n j}, \boldsymbol{r e a l}}$ and $\boldsymbol{I}_{\boldsymbol{i n j}, \boldsymbol{i m a g}}$, respectively) the problem can be transformed into a linear problem of dimension $2 n$ with no complex terms [18], e.g.:

$$
\begin{gathered}
v_{i, \text { real }}=\operatorname{Re}\left(\left[\boldsymbol{Y}_{\boldsymbol{i}, *}\right]^{-\mathbf{1}}\right) \cdot \boldsymbol{I}_{\text {inj,real }}-\operatorname{Im}\left(\left[\boldsymbol{Y}_{\boldsymbol{i}, *}\right]^{-\mathbf{1}}\right) \cdot \boldsymbol{I}_{\text {inj,imag }} \\
v_{i, \text { imag }}=\operatorname{Im}\left(\left[\boldsymbol{Y}_{\boldsymbol{i}, *}\right]^{-\mathbf{1}}\right) \cdot \boldsymbol{I}_{\text {inj,real }}+\operatorname{Re}\left(\left[\boldsymbol{Y}_{\boldsymbol{i}, *}\right]^{-\mathbf{1}}\right) \cdot \boldsymbol{I}_{\text {inj,imag }}
\end{gathered}
$$

For simplicity, complex notation will be used, and where appropriate the equations described by complex variables will be separated into real and imaginary parts.

The voltage vector $\boldsymbol{v}$ is now the direct solution to the network given Assumptions 1 and 2. As the objective is to minimize line losses, we must first calculate the current through each branch and then use this to calculate the active power losses. The current through a branch connecting nodes $i$ and $j$ is then:

$$
I_{i j}=Y_{i j}\left(v_{i}-v_{j}\right)
$$

where $Y_{i j}$ is the complex admittance of the branch, and $v_{i}$ and $v_{j}$ are the complex voltages at nodes $i$ and $j$ respectively. We define $v_{i j}$ as the complex voltage drop $\left(v_{i}-v_{j}\right)$ over a branch. We know $Y_{i j}=G_{i j}+j B_{i j}$, where $G_{i j}$ and $B_{i j}$ are the branch conductance and susceptance respectively. Since power loss depends on the magnitude of the current squared (as it is proportional to the power loss), we can write:

$$
\begin{gathered}
\left|I_{i j}\right|=\left|Y_{i j}\right|\left|v_{i j}\right|= \\
\sqrt{\left({G_{i j}}^{2}+B_{i j}{ }^{2}\right)} \sqrt{\left(v_{i, \text { real }}-v_{j, \text { real }}\right)^{2}+\left(v_{i, \text { imag }}-v_{j, \text { imag }}\right)^{2}} \\
\left|I_{i j}\right|^{2}=\left({G_{i j}}^{2}+{B_{i j}}^{2}\right)\left[\left(v_{i, \text { real }}-v_{j, \text { real }}\right)^{2}+\left(v_{i, \text { imag }}-\right.\right. \\
\left.\left.v_{j, \text { imag }}\right)^{2}\right]
\end{gathered}
$$

Since $\left(G_{i j}{ }^{2}+B_{i j}{ }^{2}\right)$ is a constant and all $v_{i, \text { real }}$ and $v_{i, i m a g}$ are linear functions of the decision variables, equation (8) is convex as it is a sum of squared linear functions. Hence this part of the objective function is convex as the power losses are equal to the squared current multiplied by the constant resistance $R_{i j}$. This can be formulated using matrix algebra, as each branch current is the difference in voltages at both ends of the line multiplied by the admittance (equation (6)). Therefore, a mapping matrix $[\boldsymbol{M}]$ can be constructed such that:

$$
I_{\text {branch }}=[M] v
$$

where $\boldsymbol{I}_{\text {branch }}$ is a $l \times 1$ vector containing all the branch currents, $[\boldsymbol{M}]$ is the $l \times n$ mapping matrix, and $\boldsymbol{v}$ is an $n \times 1$ vector containing the voltage at each node calculated by equation (3). The mapping matrix $[\boldsymbol{M}]$ relates the branch currents to the nodal voltages. Since, by Ohm's Law, the current through a branch is equal to the voltage difference at its end nodes divided by the constant impedance, it is possible to write $l$ equations which define the branch currents as linear functions of the $n$ nodal voltages. Given a branch $k$ connecting nodes $i$ and $j$, the matrix $[\boldsymbol{M}]$ has therefore the following entries:

$$
M_{k, i}=Y_{i j} \text { and } M_{k, j}=-Y_{i j}
$$

Every branch of the network is represented in this way, and all other entries of $[\boldsymbol{M}]$ are zero. This matrix is also sometimes known as a connection matrix or a branch-node incidence matrix. The power loss in the lines is a scalar $P_{\text {loss }}$ :

$$
P_{\text {loss }}=\left[\boldsymbol{I}_{\text {branch }} * \boldsymbol{I}_{\text {branch }}\right]^{\boldsymbol{T}} \cdot \boldsymbol{R}
$$

where $\boldsymbol{R}$ is a $l \times 1$ vector containing the branch resistances. Substituting (3) and (9) into (10), we have:

$$
P_{\text {loss }}=\left[\left[\boldsymbol{M}[\boldsymbol{Y}]^{-\mathbf{1}} \boldsymbol{I}_{\text {inj }}\right] *\left[\boldsymbol{M}[\boldsymbol{Y}]^{-\mathbf{1}} \boldsymbol{I}_{\text {inj }}\right]\right]^{T} \cdot \boldsymbol{R}
$$

where $*$ denotes element-wise multiplication and $\cdot$ is the dot product. Transformer losses are represented by augmenting the matrix $[\mathbf{M}]$ with an additional branch, where the additional entries in $[\mathbf{M}]$ are derived from the transformer admittance. The vector $\boldsymbol{R}$ is also augmented with the transformer resistance. Care must be taken to refer these constant values to the same side of the transformer.

Finally, the loss associated with the efficiency of each ESS must be considered. We assume that this can be modelled adequately with a one-way efficiency. Although the true efficiency depends on several time-varying factors such as the state-of-charge, a one-way efficiency approximation is usually a reasonable one. The formulation is extended to consider dissimilar charging and discharging efficiencies for each energy storage system as shown in Appendix B. Using a one-way efficiency approximation, the energy storage loss term is:

$$
E S S_{\text {loss }}=\sum_{x=1}^{s}\left(1-\eta_{x}\right)\left({\sqrt{c_{x, \text { real }}^{2}+c_{x, \text { reactive }}}}^{2}\right)
$$

where $c_{x, \text { real }}$ denotes the real power injected by the ESS $x$, $c_{x, \text { reactive }}$ denotes the reactive power injected by the ESS $x$, and $\eta_{x} \in(0,1)$ is the efficiency of a particular ESS $x$. Even though the square root operator is not a convex function, the decision variables within are squared. Hence this term takes the form of a two-norm, which is a convex function [35].

In the case of four-wire distribution systems with PME (Protective Multiple Earthing) / MEN (Multiple-EarthedNeutral) earthing systems, the loss through the neutral to earth connections $\left(N E_{\text {loss }}\right)$ should also be added:

$$
N E_{\text {loss }}=\sum_{i \in N} \frac{v_{i} \cdot v_{i}^{*}}{R_{N, i}}=\sum_{i \in N} \frac{v_{i, r e a l}{ }^{2}+v_{i, i m a g}{ }^{2}}{R_{N, i}}
$$

where $N$ describes the set of nodes on the neutral which are connected to earth, $v_{i}$ is the complex voltage at each node $i$ as before, and $R_{N, i}$ is the constant resistance between each individual neutral node and earth. This is also a convex term as both $v_{i \text {,real }}$ and $v_{i, \text { imag }}$ are linear functions of the decision 
variables. The total power loss, which is the objective function, is now:

$$
\begin{gathered}
f(c)=P_{\text {loss }}+E S S_{\text {loss }}+N E_{\text {loss }} \\
f(c)=\left[\left[\boldsymbol{M}[\boldsymbol{Y}]^{-\mathbf{1}} \boldsymbol{I}_{\boldsymbol{i n j}}\right] *\left[\boldsymbol{M}[\boldsymbol{Y}]^{-\mathbf{1}} \boldsymbol{I}_{\boldsymbol{i n j}}\right]\right]^{T} \cdot \boldsymbol{R} \\
+\sum_{x=1}^{s}\left(1-\eta_{x}\right)\left(\sqrt{c_{x, \text { real }}{ }^{2}+c_{x, \text { reactive }}}\right) \\
+\sum_{i \in N} \frac{v_{i} \cdot v_{i}{ }^{*}}{R_{N, i}}
\end{gathered}
$$

\section{Constraints}

We will assume that the operation of the ESS optimization problem occurs at a time-frame slow enough that dynamic limits (e.g. rate of change limits in ESSs) can be ignored. Consider then the scenario where a centralized controller demands a total injection of $c_{t p, \varphi}$ Watts (W) and $c_{t q, \varphi}$ VoltAmperes-reactive (VAr) from multiple single phase ESSs on the same phase $\varphi$ in one distribution network. The most obvious constraint is that the total ESS active power contribution for each phase must equal that ordered by a central controller:

$$
\begin{aligned}
& g_{1 a}(\boldsymbol{c})=\left(\sum_{x, \text { such that } \phi(x) \in A} c_{x, \text { real }}\right)-c_{t p, a}=0 \\
& g_{1 b}(\boldsymbol{c})=\left(\sum_{x, \text { such that } \phi(x) \in B} c_{x, \text { real }}\right)-c_{t p, b}=0 \\
& g_{1 c}(\boldsymbol{c})=\left(\sum_{x, \text { such that } \phi(x) \in C} c_{x, \text { real }}\right)-c_{t p, c}=0
\end{aligned}
$$

If three-phase ESSs exist, they may be represented as three single-phase ESSs, with additional constraints if the threephase ESS must be balanced:

$$
\begin{aligned}
c_{x, \text { real }} & =c_{y, \text { real }}=c_{z, \text { real }} \\
c_{x, \text { reactive }} & =c_{y, \text { reactive }}=c_{z, \text { reactive }}
\end{aligned}
$$

where $x, y$, and $z$ represent the equivalent single-phase ESSs.

\section{1) Branch capacity}

An inequality constraint is enforced on the branch currents (equation (8)) to ensure that branches are not overloaded:

$$
\left|I_{i j}\right|^{2} \leq{\overline{I_{i j}}}^{2}
$$

where $\overline{I_{i j}}$ is the current limit of the branch connecting nodes $i$ and $j$. This inequality constraint is enforced for all $l$ branches.

\section{2) Voltage limits}

To enforce maximum voltage limits, the following inequality constraints are required for all phase nodes:

$$
v_{i, \text { real }}{ }^{2}+v_{i, \text { imag }}{ }^{2} \leq{\overline{V_{i}}}^{2} \text {, for all } i \text { such that } i \notin N
$$

where $\overline{V_{i}}$ is the maximum allowable steady-state voltage at bus $i$. Since equations (4) and (5) are linear functions of the decision variables, it follows that (20) is a convex constraint. Minimum voltage constraints are more challenging as $-v_{i, \text { real }}{ }^{2}-$ $v_{i, \text { imag }}{ }^{2}$ is a concave function of the decision variables and hence the convexity of the optimization would be lost.

${ }^{1}$ In particular, the constants $K_{1 A}, K_{2 A}$ are chosen to minimize the expression $\int_{-\pi / 18}^{\pi / 18} G(\theta)\left(K_{1 A} \cos \theta+K_{2 A} \sin \theta-1\right)^{2} d \theta$, where $G(\theta)$ is the pdf of a Gaussian
However, a linear approximation of the voltage can be used instead of the concave function [18]:

$$
\begin{aligned}
& -K_{1 A} v_{i, \text { real }}-K_{2 A} v_{i, \text { imag }} \leq-\underline{V_{i}} i \in A \\
& -K_{1 B} v_{i, \text { real }}-K_{2 B} v_{i, \text { imag }} \leq-\underline{V_{i}} i \in B \\
& -K_{1 C} v_{i, \text { real }}-K_{2 C} v_{i, \text { imag }} \leq-\underline{V_{i}} i \in C
\end{aligned}
$$

where $V_{i}$ is the minimum allowable steady-state voltage at bus $i$. Using the fact that the approximate voltage angle is known at all phase nodes, the constants $K_{1 A}, K_{2 A}, K_{1 B}$, $K_{2 B}, K_{1 C}, K_{2 C}$ can be chosen such to minimize the weighted average squared approximation error over an angle deviation of $\pm 10^{\circ}$ from the approximate voltage angle. ${ }^{1}$ Since the voltage angle is likely to be very close to the expected angle, a Gaussian probability distribution function with a mean at the nominal angle and standard deviation of $2.5^{\circ}$ is used to weight the error curve. The weighted mean squared error achieved over the applicable region is $0.0039 \%$.

TABLE I. LINEAR APPROXIMATION CONSTANTS FOR EXPECTED PHASE

\begin{tabular}{c|c|c|c|c|c}
\multicolumn{7}{|c}{ ANGLES OF $0^{\circ},-120^{\circ}, 120^{\circ}$} \\
$\boldsymbol{K}_{\mathbf{1 A}}$ & $\boldsymbol{K}_{\mathbf{2 A}}$ & $\boldsymbol{K}_{\mathbf{1 B}}$ & $\boldsymbol{K}_{\mathbf{2} \boldsymbol{B}}$ & $\boldsymbol{K}_{\mathbf{1} \boldsymbol{C}}$ & $\boldsymbol{K}_{\mathbf{2} \boldsymbol{C}}$ \\
\hline \hline 1.001 & 0 & -0.5005 & -0.8668 & -0.5005 & 0.8668
\end{tabular}

Many utilities have limits on the allowable voltage unbalance factor, where the true definition of the voltage unbalance factor at bus $m\left(V U F_{m}\right)$ is the negative sequence voltage divided by the positive sequence [36]:

$$
V U F_{m}=\frac{V n_{m}}{V p_{m}}=\frac{V_{a, m}+\alpha^{2} V_{b, m}+\alpha V_{c, m}}{V_{a, m}+\alpha V_{b, m}+\alpha^{2} V_{c, m}}
$$

where $V_{a, m}$ is the phase $a$ voltage at bus $m, V_{b, m}$ and $V_{c, m}$ are the other phase voltages, $V n_{m}$ is the negative sequence voltage at bus $\mathrm{m}, V p_{m}$ is the positive sequence voltage at bus $m$ and $\alpha=$ $1 \angle 120^{\circ}=-\frac{1}{2}+j \frac{\sqrt{3}}{2}$. Although both the negative and positive sequence voltages are linear combinations of the decision variables (from equations (4) and (5)), the ratio of the two is not convex. Hence, the following approximation is proposed:

$$
V U F_{m}=\frac{V n_{m}}{V p_{m}} \cong \frac{V_{a, m}+\alpha^{2} V_{b, m}+\alpha V_{c, m}}{V_{\varphi}}
$$

where $V_{\varphi}$ is the nominal, estimated or measured network voltage at phase $\varphi$. This is a very good approximation as in practice the positive sequence voltage should always be much larger than the negative sequence and close to the nominal voltage. Hence the voltage unbalance factor is now a linear function of the decision variables. The inequality constraint is:

$$
V U F_{m}{ }^{2} \leq{\overline{V U F_{m}}}^{2} \text {, for all buses } m
$$

where $\overline{V U F_{m}}$ is the maximum allowable voltage unbalance factor at bus $m$. Finally, some countries or utilities require the magnitude of the neutral voltage displacement to be below a specified maximum voltage $\overline{V_{\text {neutral }}}$. This inequality constraint is convex: 


$$
v_{i, \text { real }}{ }^{2}+v_{i, \text { imag }}{ }^{2} \leq{\overline{V_{\text {neutral }}}}^{2}, i \in N
$$

3) ESS limits

ESS size and power constraints are considered by enforcing:

$$
c_{x, \text { real }}{ }^{2}+c_{x, \text { reactive }}{ }^{2} \leq{\overline{c_{x}}}^{2} \text { for all ESS } x
$$

where $\overline{c_{x}}$ is the rating of the inverter connecting the ESS to the grid. The state-of-charge at $\operatorname{ESS} x\left(S_{0} C_{x}\right)$ also constrains the active power which may be supplied to the network or sunk from the network. If the state-of-charge is defined as being between 0 and 1 , where 0 represents a fully discharged ESS and 1 represents a fully charged ESS, the constraints are:

$$
\begin{aligned}
-c_{x, \text { real }} & \leq \frac{E_{\text {capacity }, x}}{\Delta t}\left(\overline{\text { SoC }_{x}}-S_{\text {So }}\right) \\
c_{x, \text { real }} & \leq \frac{E_{\text {capacity }, x}}{\Delta t}\left(\operatorname{SoC}_{x}-\underline{S o C_{x}}\right)
\end{aligned}
$$

where $S o C_{x}$ and $\overline{S o C_{x}}$ are the minimum and maximum allowable state-of-charge at ESS $x$, and $\Delta t$ is the time granularity. In Appendix A we extend this to consider multiperiod energy transition in a time scheduling problem. Note that the framework can also consider controllable loads in a similar way via a suitable cost function to be added to the objective and minimum and maximum power constraints.

\section{Full definition}

The full definition of the optimization problem considered is:

$$
\begin{aligned}
& \text { minimize } \\
& f(c)=\left[\left[\boldsymbol{M}[\boldsymbol{Y}]^{-1} \boldsymbol{I}_{i n j}\right] *\left[\boldsymbol{M}[\boldsymbol{Y}]^{-1} \boldsymbol{I}_{i n j}\right]\right]^{T} \cdot \boldsymbol{R}
\end{aligned}
$$

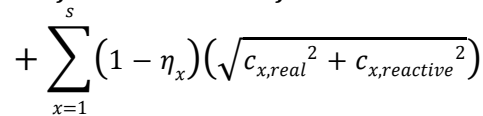

$$
\begin{aligned}
& \sum_{i \in N}^{x=1} \frac{v_{i} \cdot v_{i}{ }^{*}}{R_{N, i}} \\
& \text { with respect to: } \\
& c_{x, \text { real }}, c_{x, \text { reactive }} \text { for all ESSs } x
\end{aligned}
$$

subject to

$$
\begin{aligned}
& g_{1 a}(\boldsymbol{c})=\left(\sum_{x, \text { such that } \phi(x) \in A} c_{x, \text { real }}\right)-c_{t p, a}=0 \\
& g_{1 b}(\boldsymbol{c})=\left(\sum_{x, \text { such that } \phi(x) \in B} c_{x, \text { real }}\right)-c_{t p, b}=0 \\
& g_{1 c}(\boldsymbol{c})=\left(\sum_{x, \text { such that } \phi(x) \in C} c_{x, \text { real }}\right)-c_{t p, c}=0 \\
& \left|I_{i j}\right|^{2} \leq{\overline{I_{i j}}}^{2} \text { for all branches connecting nodes }(i, j) \\
& v_{i, \text { real }}{ }^{2}+v_{i, \text { imag }}{ }^{2} \leq{\overline{V_{i}}}^{2} \text {, for all } i, i \notin N \\
& -K_{1 A} v_{i, \text { real }}-K_{2 A} v_{i, \text { imag }} \leq-V_{i} i \in A \\
& -K_{1 B} v_{i, \text { real }}-K_{2 B} v_{i, \text { imag }} \leq-\bar{V}_{i} i \in B \\
& -K_{1 C} v_{i, \text { real }}-K_{2 C} v_{i, i m a g} \leq-\underline{V}_{i} i \in C \\
& V U F_{m}{ }^{2} \leq{\overline{V U F_{m}}}^{2} \text {, for all buses } m \\
& v_{i, \text { real }}{ }^{2}+v_{i, \text { imag }}{ }^{2} \leq{\overline{V_{\text {neutral }}}}^{2}, i \in N \\
& c_{x, \text { real }}{ }^{2}+c_{x, \text { reactive }}{ }^{2} \leq{\overline{c_{x}}}^{2} \text { for all ESSs } x \\
& -c_{x, \text { real }} \leq \frac{E_{\text {capacity, } x}}{\Delta t}\left(\overline{\text { SoC }_{x}}-S_{\text {o }} C_{x}\right) \\
& c_{x, \text { real }} \leq \frac{E_{\text {capacity }, x}}{\Delta t}\left(\operatorname{SoC}_{x}-\underline{\operatorname{So} C_{x}}\right)
\end{aligned}
$$

Assuming normal network operation is feasible, an optimal solution exists if the magnitude of each $c_{t p, \varphi}$ is not unfeasibly large. Very large $c_{t p, \varphi}$ values may unavoidably constrain line, voltage or ESS power rating limits.

Remark 3: Note that a practical implementation of this optimized ESS dispatch algorithm would also require the issue of scheduling to be addressed since the ESS limits depending on the state-of-charge are influenced by decisions in past time periods. This extension is addressed in Appendix A.

\section{CASE STUDIES AND DISCUSSION}

The case studies are performed on a representative urban fourwire unbalanced distribution network in New Zealand which supplies 71 customers. The network data and sample load profiles are available in [37] and the supplementary file ${ }^{2}$. The neutral conductor is explicitly modelled in the simulation, and it is earthed at multiple points in what is variously known as a Terrestrial Neutral Combined and Separated (TN-C-S) system, MEN, or a PME system. The single line diagram of the test network is shown in Fig. 2, and the total load profile for each phase is shown in Fig. 3.

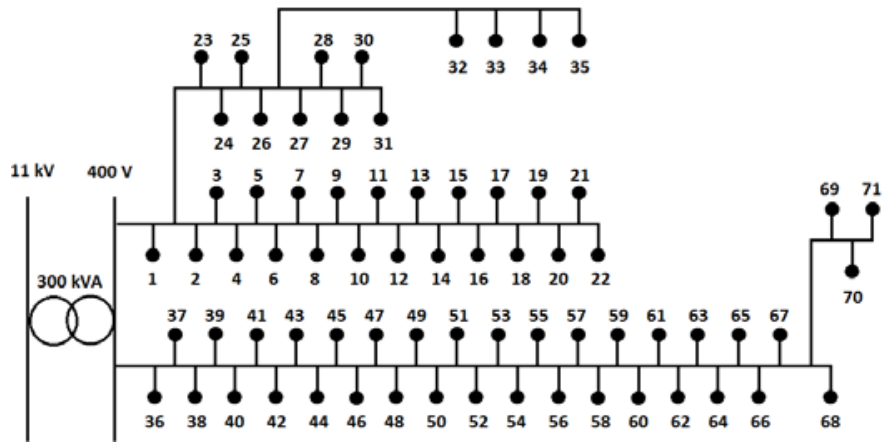

Fig. 2. Topology of the test network
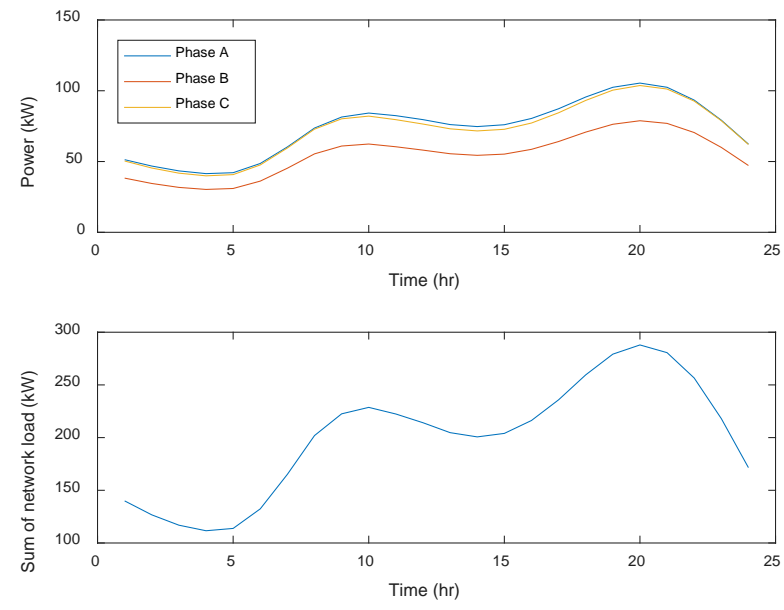

Fig. 3. Network load profiles

\section{A. Power-flow comparison}

Part A of the case study compares the reduced approximate power-flow used by the convex optimization formulation derived in this paper to a full power-flow. Both the reduced power-flow and the full non-linear power flow are implemented in a custom MATLAB solver which was developed and verified against PSS SINCAL and Simscape Power Systems in [15]. 
The proposed model considers constant current and constant impedance loads only. While this is likely to be a reasonable assumption in many distribution networks, in this case study we have taken the worst case (all loads are constant power) when comparing the proposed approximate power-flow to the full power-flow. Hence, the approximation error includes the error arising from this simplification.

Fig. 4 demonstrates the very good accuracy of the approximate power-flow by comparing the predicted voltages and currents to the full AC power-flow at peak load without ESS contribution, plotting the distribution of the voltage and current error. This simulation uses a preliminary estimation of the network voltages as mentioned in Remark 2. A flat initial voltage profile results in larger, but still acceptable, errors of up to $2 \times 10^{-3} \mathrm{~V}$ (p.u.) and $2 \%$ current respectively. A further comparison is given in Fig. 5 which compares the predicted power losses (including losses in the neutral) against the reference (full AC power-flow) losses in the test network at each hour of the day.
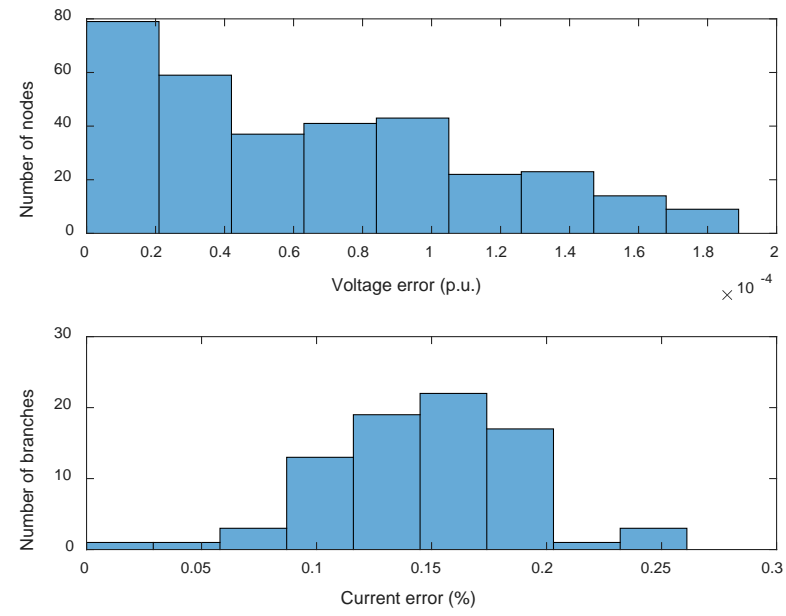

Fig. 4. Histogram of voltage and current errors in the test network

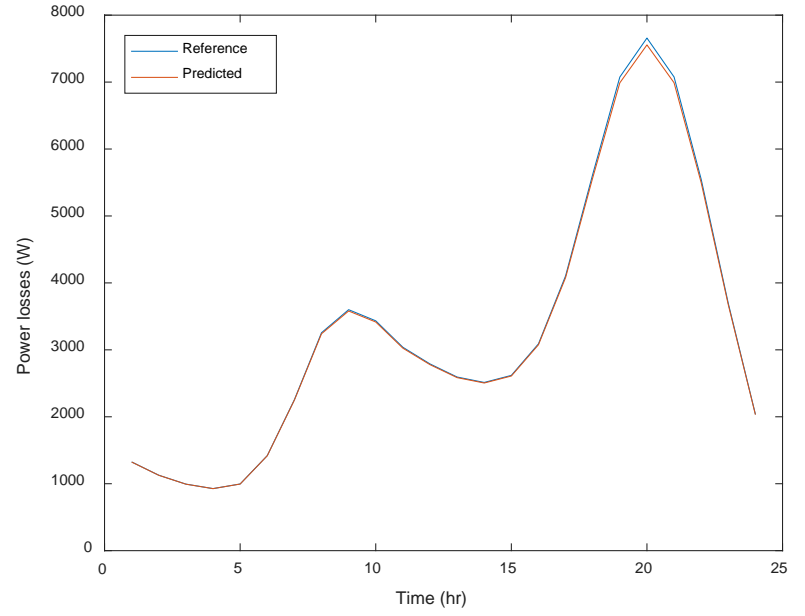

Fig. 5. Comparison of network losses in the approximate formulation ("Predicted") and in the full AC power-flow ("Reference")

\section{B. Losses in the neutral}

The proportion of losses in the neutral depend primarily on the load unbalance of the network, the earthing system, the distribution of the unbalanced connections, and the impedance of the neutral conductor [30]. Load unbalance is defined as the maximum deviation between phase powers divided by the average. The neutral loss percentage is important for deciding when the explicit representation of the neutral wire should be used. Fig. 6 shows the effect of load unbalance on the neutral losses in the test system for various earthing systems and neutral conductor impedances. The results in Fig. 6a are comparable with the result in [38] for $8 \%$ load unbalance.

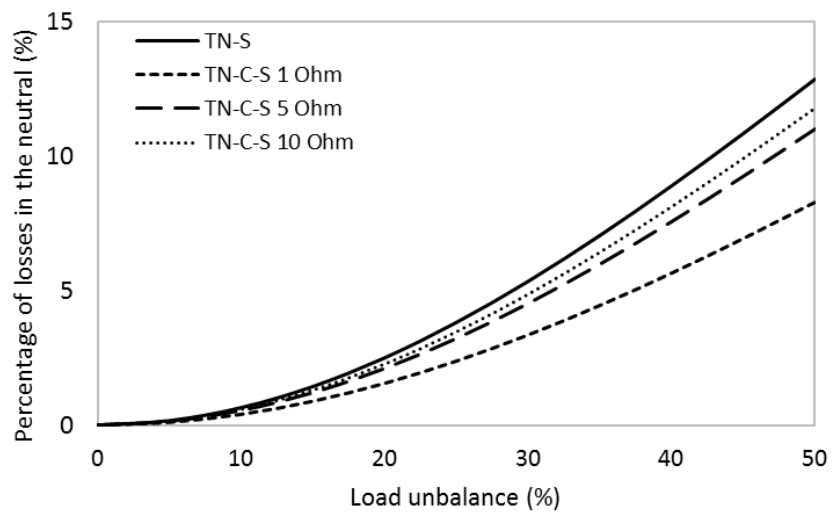

a) Assuming neutral impedance is equal to phase impedance

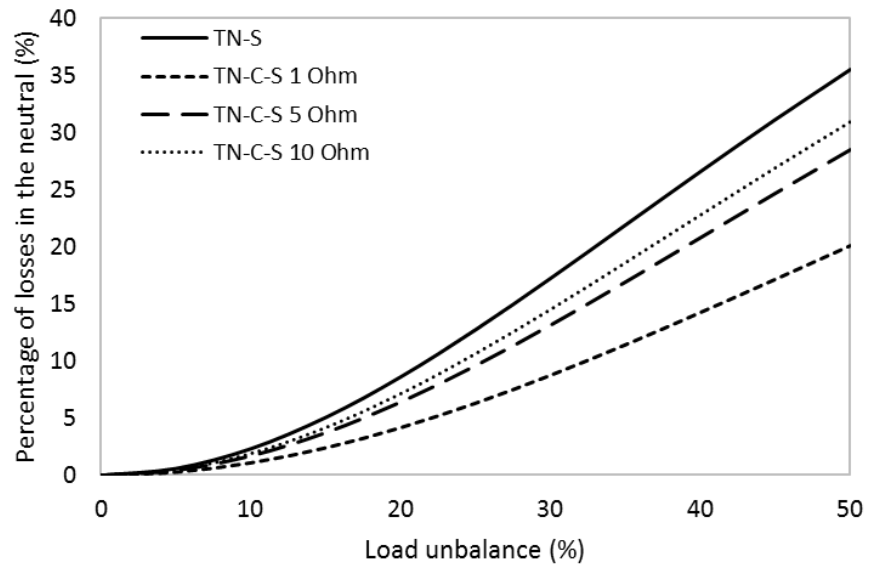

b) Assuming neutral impedance is twice the phase impedance

Fig. 6. Effect of load unbalance on the neutral losses for various earthing systems

Fig. 7 shows the effect of the neutral wire impedance on the neutral losses for various earthing systems and 20\% load unbalance. The $1 \mathrm{Ohm}, 5 \mathrm{Ohm}$ and $10 \mathrm{Ohm}$ curves represent the individual resistive impedance of the neutral-to-earth connection at each load. It is evident that when the load unbalance is high, it is important to consider the neutral wire explicitly. The loss percentage in the neutral is often higher than the approximation error, especially for the Terrestrial Neutral Separated (TN-S) system or higher impedance neutral conductors. 


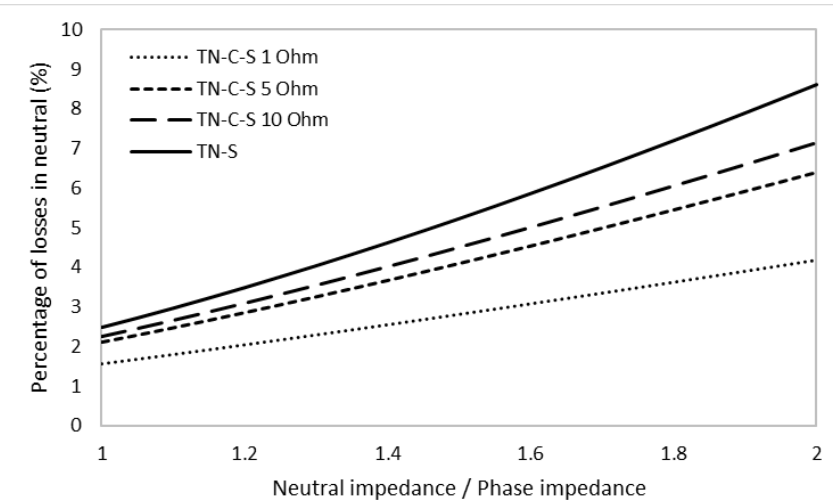

Fig. 7. Effect of the neutral wire impedance on the neutral losses (20\% load unbalance)

\section{Reduction of total losses and OPF comparison}

Using the daily load profiles at each load node, the energy saved by the proposed optimized method is demonstrated. Ten ESSs are simulated in the test network, the locations being randomly chosen from equiprobable load nodes; and they aggregately supply or sink power to flatten the network load profile. The one-way efficiency is 0.9 or $90 \%$, and the nominal voltage is $230 \mathrm{~V}$ phase-to-neutral with voltage limits of $\pm 6 \%$ p.u. and the transformer tap is set to 1.04 p.u. The base scenario is equitable contributions from each ESS, which is the starting point for the optimization problem, and a flat initial voltage profile is used. The load profiles have a time resolution of 1 hour, and the convex approximate OPF formulated in section III is solved at each hour by the interior-point solver fmincon in MATLAB. The simulation results are given in Fig. 8. By evaluating the area between the two curves, the total losses are calculated to be reduced by $14.08 \%$ overall. The ESS losses do not vary significantly as all ESS units have the same efficiency; however, the line losses are reduced by $33.20 \%$.

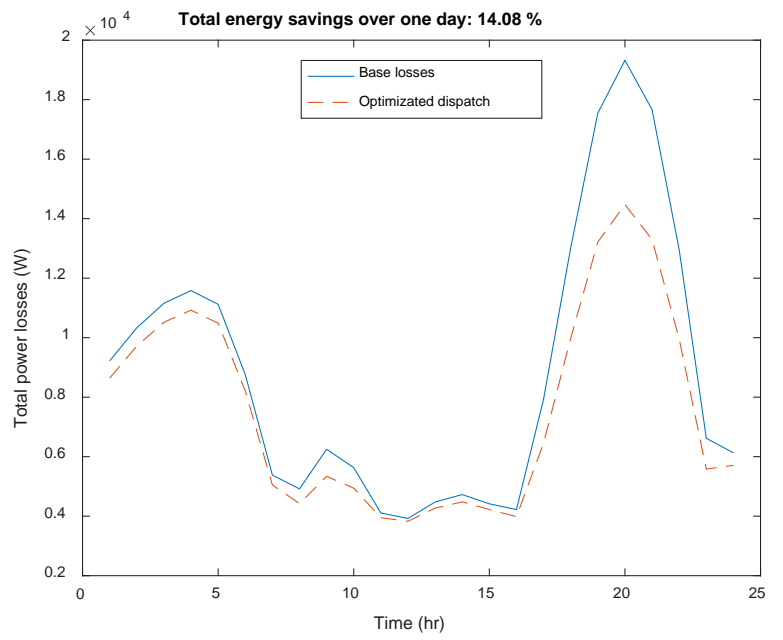

Fig. 8. Demonstration of the power loss reduction throughout one day

The simulations in Fig. 8 assumed that all ESS storage systems in the distribution network have identical one-way efficiencies. In practice this may not be the case. Figs. 9 and 10 show the power savings throughout the day where the efficiency of each individual ESS was randomly and independently chosen from a Gaussian distribution with mean $90 \%$ and variance $\left(\sigma^{2}\right)$ of $2 \%$ (Fig. 9 ) and $4 \%$ (Fig. 10). The one-way efficiency of each ESS is bounded between 0.825 and
0.975 since it is impossible to achieve an arbitrarily high efficiency. The energy savings are increased to $18.90 \%$ in Fig. 9 and $21.77 \%$ in Fig. 10.

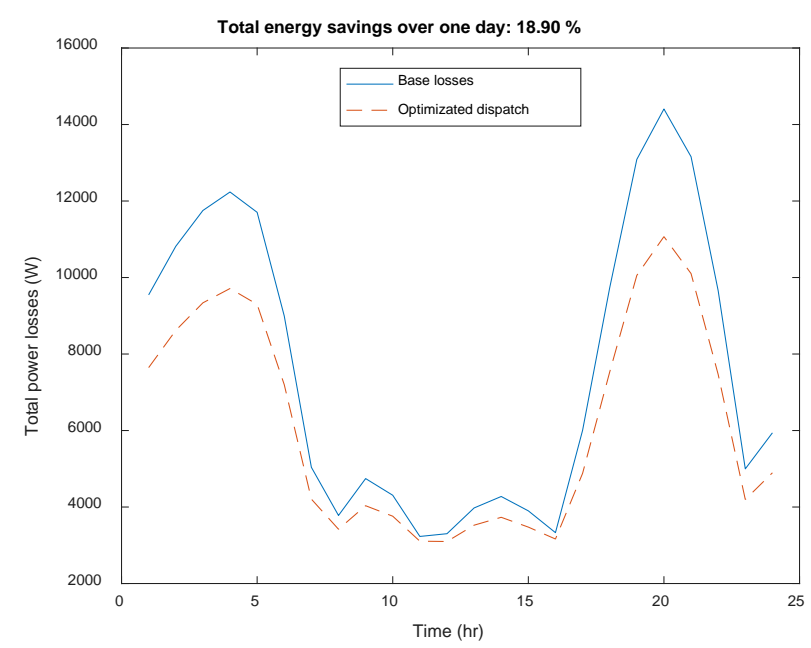

Fig. 9. Demonstration of the power loss reduction throughout one day, varied ESS efficiency $\left(\sigma^{2}=2 \%\right)$

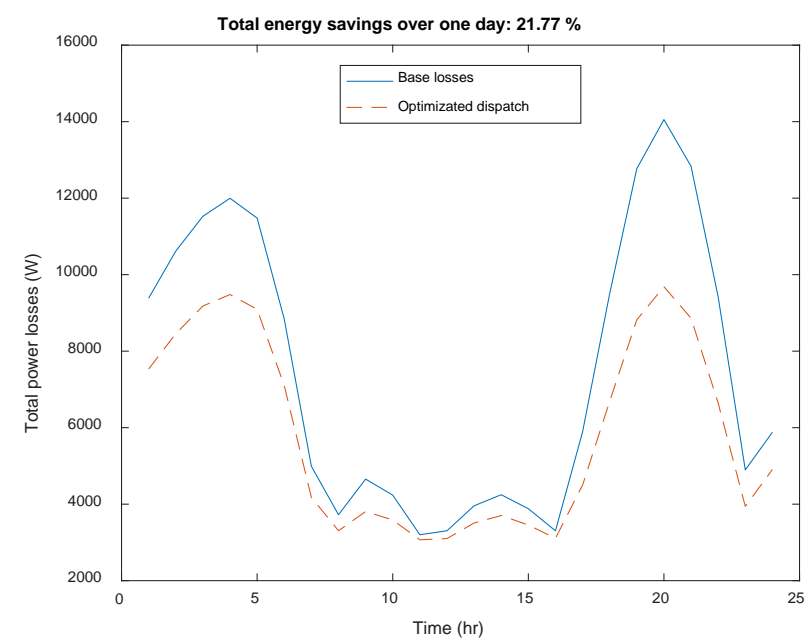

Fig. 10. Demonstration of the power loss reduction throughout one day, varied ESS efficiency $\left(\sigma^{2}=4 \%\right)$

To demonstrate that the proposed formulation is effective in a meshed network, the ends of the two feeders are connected to a new distribution transformer secondary (identical to the existing one) via hypothetical low impedance links of $0.001+$ $\mathrm{j} 0.001 \Omega$ in simulation, as illustrated in Fig. 11. Fig. 12 shows the simulation result for the meshed network.

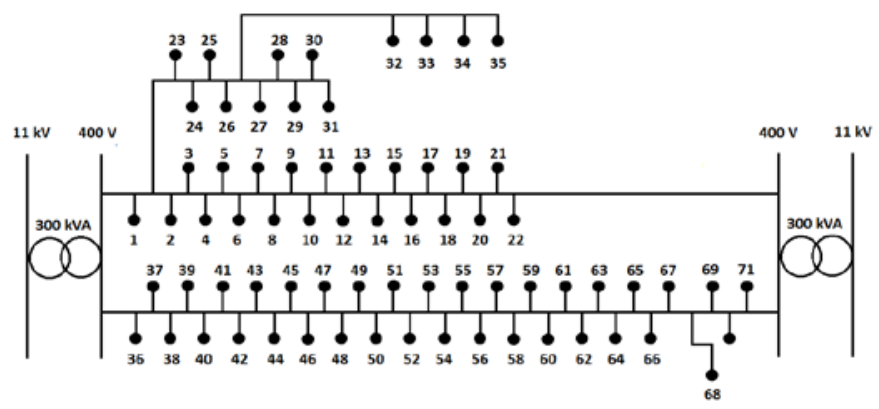

Fig. 11. Topology of the meshed network 


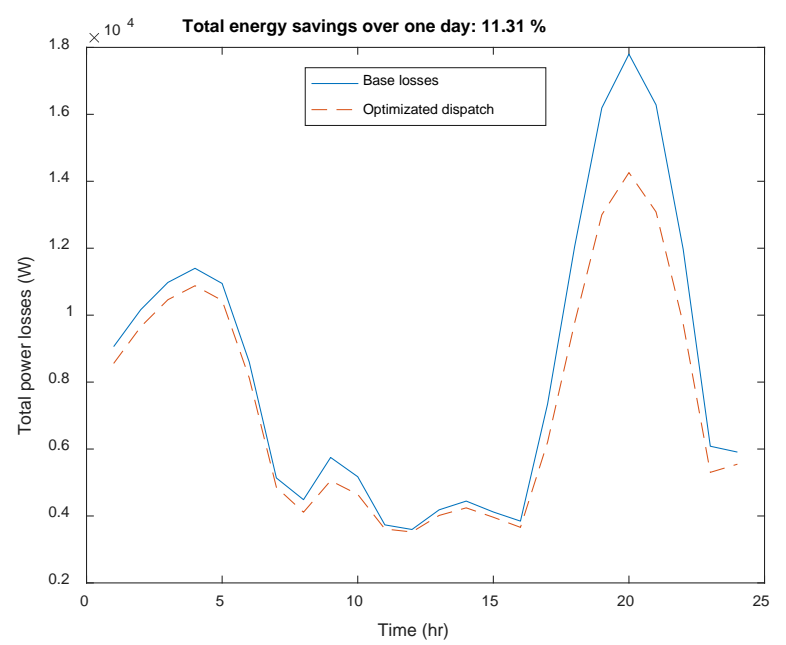

Fig. 12. Demonstration of the power loss reduction throughout one day for the simulated meshed network

The proposed method was also compared to the related nonlinear and non-convex $\mathrm{AC} \mathrm{OPF}^{3}$. Table II shows the optimality ratio statistics for the studies in this section, where the optimality gap is defined as the difference between the value of the true objective function at the approximate solution of the proposed method and the non-convex AC OPF, normalized with the value of the true objective function at the solution of the non-convex AC OPF. The maximum optimality gap refers to the maximum value of the optimality gap at any time during the simulated day as presented in Figs. 8-10 and 12.

TABLE II. OPTIMALITY STATISTICS IN THE CASE STUDIES

\begin{tabular}{|c|c|c|c|c|}
\hline Case & Network & $\begin{array}{c}\text { ESS efficiency } \\
\text { variance }\end{array}$ & $\begin{array}{c}\text { Maximum } \\
\text { optimality gap }\end{array}$ & $\begin{array}{c}\text { Average } \\
\text { optimality gap }\end{array}$ \\
\hline $\mathbf{1}$ & Radial & $0 \%$ & $0.07 \%$ & $0.01 \%$ \\
\hline $\mathbf{2}$ & Radial & $2 \%$ & $0.35 \%$ & $0.03 \%$ \\
\hline $\mathbf{3}$ & Radial & $4 \%$ & $0.93 \%$ & $0.15 \%$ \\
\hline $\mathbf{4}$ & Meshed & $0 \%$ & $0.02 \%$ & $0.00 \%$ \\
\hline
\end{tabular}

\section{Computational time}

In order to demonstrate the practical advantages of the convex formulation in terms of scaling, the computational time performance of the proposed algorithm was compared to the non-convex AC OPF with a convergence tolerance of $10^{-2} \mathrm{~V}$ at any node for the associated non-linear power-flow. Both algorithms were executed in MATLAB on an Intel ${ }^{\circledR}$ Xeon ${ }^{\circledR}$ CPU E3-1240 V2 @ 3.40 GHz.

Monte-Carlo simulations (50 for each data point) were required as the optimization time in this problem depends on the starting point and other unpredictable factors. Due to the presence of multiple phases, if unbalance constraints are not active the total optimization time is likely to depend mostly on how many ESSs are on the phase with the most ESS connections. The test network is the radial network of Fig. 2, and the total ESS active power was set to 1000W / ESS for each phase. Fig. 1 shows that the proposed algorithm scales much better than the non-convex AC OPF.

\footnotetext{
${ }^{3}$ The non-convex AC OPF was solved by taking the minimum feasible value found by any of the MATLAB functions ga (genetic algorithm), pso (particle
}

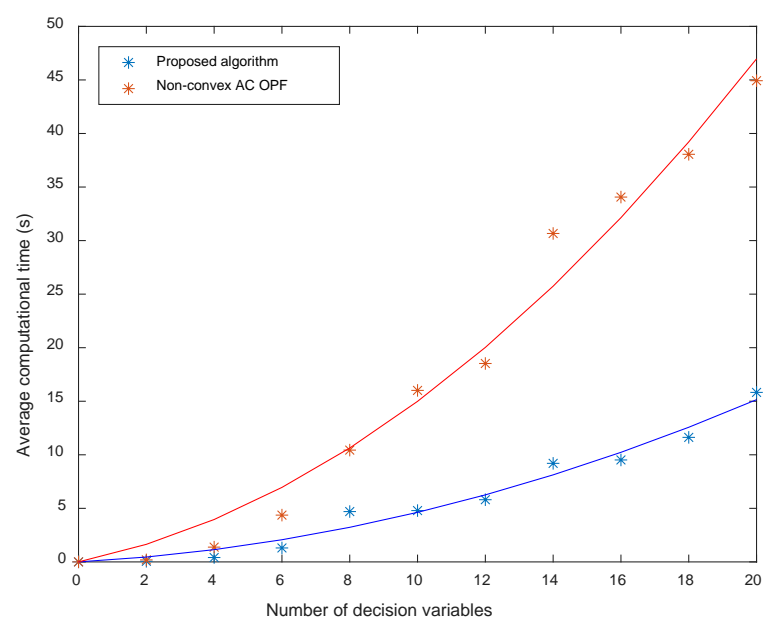

Fig. 13. Computational time comparison

\section{CONCLUSION}

This paper has presented a convex approximate AC optimal power-flow for unbalanced three-phase four-wire distribution networks with energy storage systems. The problem is of practical importance, as previous literature on convexified optimal power flow has not considered unbalanced effects and constraints in detail. Furthermore, we have extended the formulation to consider ESS time scheduling, and the application of network unbalance constraints to this problem is also novel.

Several challenges exist which are unique to unbalanced networks, and we have made assumptions in order to be able to incorporate unbalance constraints into the convex approximate power-flow. These assumptions have been substantiated by reference to the features of typical distribution networks, and the accuracy of the formulation was demonstrated in an actual distribution system.

Consideration was also given to the losses and voltages in the neutral wire. The factors which determine when it becomes important to consider neutral losses were identified and the relationship explored. Furthermore, it was shown that losses in the neutral wire can often be significant; and hence it is important to consider this in the power allocation problem. Finally, the practical energy saving of the proposed framework was demonstrated in several test cases.

\section{REFERENCES}

[1] H. Farhangi, "The path of the smart grid," IEEE Power \& Energy Magazine, vol. 8, no. 1, pp. 18-28, 2010.

[2] P. Denholm, E. Ela, B. Kirby and M. Milligan, "The Role of Energy Storage with Renewable Electricity generation," NREL, 2010.

[3] X. Li, D. Hui and X. Lai, "Battery Energy Storage Station (BESS)Based Smoothing Control of Photovoltaic (PV) and Wind Power Generation Fluctuations," IEEE Transactions on Sustainable Energy, vol. 4, no. 2, pp. 464 - 473, 2013.

[4] M. M. Haque and P. Wolfs, "A review of high PV penetrations in LV distribution networks: Present status, impacts and mitigation measures,"

swarm optimization), patternsearch, or fmincon with the interior-point optimizer starting at the solution of the convex optimization problem. 
Renewable and Sustainable Energy Reviews, vol. 62, pp. 1195-1208, 2016.

[5] T. Hong and J. Burke, "Calculating Line Losses in Smart Grid: A New Rule of Thumb," in Transmission and Distribution Conference and Exposition, 2010 IEEE PES, 2010.

[6] Office of Gas and Electricity Markets (Ofgem), "Electricity distribution losses: a consultation document," Ofgem, London, UK, 2003.

[7] O. Palizban and K. Kauhaniemi, "Distributed cooperative control of battery energy storage system in AC microgrid applications," Journal of Energy Storage, vol. 3, pp. 43-51, 2015.

[8] Z. Yang, R. Wu, J. Yang, K. Long and P. You, "Economical operation of microgrid with various devices via distributed optimization," IEEE Transactions on Smart Grid, vol. 7, no. 2, p. 857-867, 2016.

[9] Y. Zhang, N. Rahbari-Asr, J. Duan and M. Chow, "Day-Ahead Smart Grid Cooperative Distributed Energy Scheduling With Renewable and Storage Integration," IEEE Transactions on Sustainable Energy, vol. 7, no. 4, pp. 1739 - 1748, 2016.

[10] K. Baker, J. Guo, G. Hug and X. Li, "Distributed MPC for efficient coordination of storage and renewable energy sources across control areas," IEEE Transactions on Smart Grid, vol. 7, no. 2, pp. 992-1001, 2016.

[11] D. Q. Hung, N. Mithulananthan and R. Bansal, "Integration of PV and BES units in commercial distribution systems considering energy loss and voltage stability," Applied Energy, vol. 113, pp. 1162-1170, 2014.

[12] W. Hu, Z. Chen, B. Bak-Jensen and Y. Hu, "Fuzzy adaptive particle swarm optimisation for power loss minimisation in distribution systems using optimal load response," IET Generation, Transmission \& Distribution, vol. 8, no. 1, pp. 1-10, 2013.

[13] Open DSS, "Open DSS Manual," 2016. [Online]. Available: https://sourceforge.net/p/electricdss/code/HEAD/tree /trunk/Distrib/Doc/OpenDSSManual.pdf.

[14] N. Yang, "Three-phase power flow calculations using direct Z BUS method for large-scale unbalanced distribution networks," IET Generation, Transmission \& Distribution, vol. 10, no. 4, pp. 10481055, 2016.

[15] J. D. Watson, N. R. Watson, D. Santos-Martin, A. R. Wood, S. Lemon and A. J. Miller, "Impact of solar photovoltaics on the low-voltage distribution network in New Zealand," IET Generation, Transmission \& Distribution, vol. 10, no. 1, pp. 1-9, 2016.

[16] R. Jabr, "Modeling Network Losses Using Quadratic Cones," IEEE Transactions on Power Systems, vol. 20, no. 1, pp. 505-506, 2005.

[17] A. Castillo, P. Lipka, J. P. Watson, S. Oren and R. P. O'Neill, "A Successive Linear Programming Approach to Solving the IV-ACOPF," IEEE Transactions on Power Systems, vol. 31, no. 4, pp. 2752-2763, 2016.

[18] R. P. O’Neill, A. Castillo and M. Cain, "The IV Formulation and Linear Approximations of the AC Optimal Power Flow Problem," Federal Energy Regulatiuon Commission, 2012.

[19] C. Coffrin and P. V. Hentenryck, "A Linear-Programming Approximation of AC Power-flows," arXiv:1206.3614v3, 2013.

[20] J. R. M. Franco, M. Lavorato and R. Romero, "A set of linear equations to calculate the steady-state operation of an electrical distribution system," in Proc. 2011 IEEE PES Conf. Innovative Smart Grid, 2011.

[21] A. Mohapatra, P. Bijwe and B. Panigrahi, "Efficient sequential nonlinear optimal power flow approach using incremental variables," IET Generation, Transmission \& Distribution, vol. 7, no. 4, p. 14731480, 2013.

[22] T. Akbari and M. T. Bina, "Linear approximated formulation of AC optimal power flow using binary discretisation," IET Generation, Transmission \& Distribution, vol. 10, no. 5, pp. 1117-1123, 2015.

[23] H. Yuan, F. Li, Y. Wei and J. Zhu, "Novel Linearized Power Flow and Linearized OPF Models for Active Distribution Networks with Application in Distribution LMP," IEEE Transactions on Smart Grid, vol. Early Access, 2016.

[24] T. Chen and W. Yang, "Analysis of Multi-Grounded Four-Wire Distribution Systems Considering the Neutral Grounding," IEEE Transactions on Power Delivery, vol. 16, no. 4, 2001.
[25] L. Gan, N. Li, U. Topcu and S. H. Low, "Exact Convex Relaxation of Optimal Power Flow in Radial Networks," IEEE Transactions on Automatic Control, vol. 60, no. 1, pp. 72-87, 2015.

[26] C. Coffrin, H. Hijazi and P. Van Hentenryck, "The QC Relaxation: A Theoretical and Computational Study on Optimal Power Flow," IEEE Transactions on Power Systems, vol. 31, no. 4, pp. 3008-3018, 2016.

[27] Q. Peng and S. Low, "Distributed Algorithm for Optimal Power Flow on an Unbalanced Radial Network," in IEEE Conference on Decision and Control, Osaka, 2015.

[28] M. Nick, R. Cherkaoui, J.-Y. L. Boudec and M. Paolone, "An Exact Convex Formulation of Optimal Power Flow in Radial Distribution Networks Including Transverse Components," ArXiv:1605.01964, 2016.

[29] J. Franco, L. Ochoa and R. Romero, "AC OPF for smart distribution networks: An efficient and robust quadratic approach," IEEE Transactions on Smart Grid, vol. Accepted Paper, 2017.

[30] S. Pajic and A. E. Emanuel, "Effect of Neutral Path Power Losses on the Apparent Power Definitions: A Preliminary Study," IEEE Transactions on Power Delivery, vol. 24, no. 2, pp. 517-523, 2009.

[31] M. J. E. Alam, K. M. Muttaqi and D. Sutanto, "Alleviation of Neutralto-Ground Potential Rise Under Unbalanced Allocation of Rooftop PV Using Distributed Energy Storage," IEEE Transactions on Sustainable Energy, vol. 6, no. 3, pp. 889-898, 2015.

[32] W. H. Kersting, Distribution System Modeling and Analysis, Florida, USA: CRC Press, 2002.

[33] A. Ballanti and L. Ochoa, "Assessing the effects of load models on MV network losses," in Australian Universities Power Engineering Conference, 2015.

[34] R. M. Ciric, A. Padilha and L. F. Ochoa, "Power Flow in Four-Wire Distribution Networks - General Approach," IEEE Transactions on Power Systems , vol. 18, no. 4, pp. 1283-1290, 2003.

[35] S. Boyd and L. Vandenberghe, Convex Optimization, Cambridge: Cambridge University Press, 2004, p. 72.

[36] P. Pillay and M. Manyage, "Definitions of Voltage Unbalance," IEEE Power Engineering Review, pp. 50-51, 2001.

[37] J. Watson, N. Watson, D. Santos-Martin, S. Lemon, A. Wood and A. Millar, "Low Voltage Network Modelling," in EEA Conference \& Exhibition, Auckland, 2014.

[38] C. Acosta, R. A. Hincapié, M. Granada, A. H. Escobar and R. A. Gallego, "An Efficient Three Phase Four Wire Radial Power Flow Including Neutral-Earth Effect," J Control Autom Electr Syst , vol. 24, p. 690-701, 2013.

\section{APPENDIX A. EXTENSION TO MULTI-PERIOD TIME OPTIMIZATION}

Given suitable load and distributed generation forecasts, the optimal dispatch formulation (28) may be extended to a multiperiod time scheduling problem with a horizon $h$. The constraints which depend on past decisions are maximum charging and discharging capacity constraints (28.12-28.13). Let us therefore define the energy stored in each ESS at the end of each time slot $k$ as:

$$
E(x, k)=E_{0}+\sum_{r=0}^{k} E_{g}(r)-\sum_{r=0}^{k} c_{x, \text { real }}(r) \Delta t f\left(x, c_{x, \text { real }}(r)\right)
$$

where $x$ is the energy storage system in question, $E_{0}$ is the initial energy of the storage device, $E_{g}(r)$ is the energy input from any integrated distributed generator throughout the time slot $r$, $c_{x, \text { real }}(r)$ is the ESS dispatch throughout the time slot $r, \Delta t$ is the time granularity (i.e. the length of each time slot), and $f(x, c)$ is defined as: 


$$
f(x, c)=\left\{\begin{array}{l}
\eta_{x} \text { if } c<0 \\
\frac{1}{\eta_{x}} \text { if } c \geq 0
\end{array} \quad \text { where } 0<\eta_{x}<1\right.
$$

(28.12) and (28.13) are then replaced by:

$$
\begin{gathered}
E(x, k) \leq \overline{E_{x}} \text { for all ESS } x \text { and time slots } k \\
-E(x, k) \leq-\underline{E_{x}} \text { for all ESS } x \text { and time slots } k
\end{gathered}
$$

where $\underline{E_{x}}$ and $\overline{E_{x}}$ denote the minimum and maximum limits of the energy that may be stored in the ESS $x$. It is sufficient to write $E(x, k)$ as a linear function of the decision variables for the constraints above to be convex. There is a standard method to achieve this by defining two new decision variables $c_{x, \text { real }}(k)^{(+)}$and $c_{x, \text { real }}(k)^{(-)}$[9], where:

$$
\begin{aligned}
& c_{x, \text { real }}(k)^{(+)}=\left\{\begin{array}{cl}
c_{x, \text { real }}(k) & \text { if } c_{x, \text { real }}(k) \geq 0 \\
0 & \text { otherwise }
\end{array}\right. \\
& c_{x, \text { real }}(k)^{(-)}=\left\{\begin{array}{cl}
0 & \text { if } c_{x, \text { real }}(k) \geq 0 \\
-c_{x, \text { real }}(k) & \text { otherwise }
\end{array}\right.
\end{aligned}
$$

and the actual dispatch of the energy storage system is:

$$
c_{x, \text { real }}(k)=c_{x, \text { real }}(k)^{(+)}-c_{x, \text { real }}(k)^{(-)}
$$

Then we have the following linear relation:

$$
\begin{aligned}
E(x, k)=E_{0}+\sum_{r=0}^{k} & E_{g}(r) \\
& \quad-\sum_{r=0}^{k} \frac{1}{\eta_{x}} c_{x, \text { real }}(r)^{(+)} \Delta t+\sum_{r=0}^{k} \eta_{x} c_{x, \text { real }}(r)^{(-)} \Delta t
\end{aligned}
$$

At the optimum point, at least one of $c_{x \text {,real }}(k)^{(+)}$or $c_{x, \text { real }}(k)^{(-)}$is zero at each time slot $k$ if we use the definition of $c_{x, \text { real }}(k)^{(+)}$and $c_{x, \text { real }}(k)^{(-)}$to rewrite the ESS loss term (12) as:

$$
\sum_{x=1}^{s}\left(1-\eta_{x}\right)\left(\sqrt{c_{x, \text { real }}(k)^{(+)^{2}}+c_{x, \text { real }}(k)^{(-)^{2}}+c_{x, \text { reactive }}(k)^{2}}\right) .
$$

Clearly for a certain $c_{x, \text { real }}(k)$ which minimizes the objective, at least one of $c_{x, \text { real }}(k)^{(+)}$or $c_{x, \text { real }}(k)^{(-)}$must be zero. Then the full definition of the time scheduling optimization problem with horizon $h$ is:

$$
\begin{gathered}
f(c, h) \\
\text { minimize } \\
=\sum_{k=0}^{h}\left\{\left[\left[\boldsymbol{M}[\boldsymbol{Y}]^{-\mathbf{1}} \boldsymbol{I}_{\boldsymbol{i n j}}(\boldsymbol{k})\right] *\left[\boldsymbol{M}[\boldsymbol{Y}]^{-\mathbf{1}} \boldsymbol{I}_{\boldsymbol{i n j}}(\boldsymbol{k})\right]\right]^{\boldsymbol{T}} \cdot \boldsymbol{R}\right. \\
+\sum_{x=1}^{s}\left(1-\eta_{x}\right)\left(\sqrt{c_{x, \text { real }}(k)^{(+)^{2}}+c_{x, \text { real }}(k)^{(-)^{2}}+c_{x, \text { reactive }}(k)^{2}}\right) \\
\left.+\sum_{i \in N} \frac{v_{i}(k) \cdot v_{i}(k)^{*}}{R_{N, i}}\right\} \\
\begin{array}{c}
\text { with respect to: } \\
c_{x, \text { real }}(k)^{(+)}, c_{x, \text { real }}(k)^{(-)}, c_{x, \text { reactive }}(k) \\
\text { for all ESS } x \text { and time slots } k
\end{array}
\end{gathered}
$$

subject to the following constraints for all time slots $k$

$$
\begin{aligned}
& g_{1 a}(\boldsymbol{c}, k)=\left(\sum_{x, \text { such that } \phi(x) \in A} c_{x, \text { real }}(k)^{(+)}-c_{x, \text { real }}(k)^{(-)}\right)-c_{t p, a}(k)=0 \\
& g_{1 b}(\boldsymbol{c}, k)=\left(\sum_{x, \text { such that } \phi(x) \in B} c_{x, \text { real }}(k)^{(+)}-c_{x, \text { real }}(k)^{(-)}\right)-c_{t p, b}(k)=0 \\
& g_{1 c}(\boldsymbol{c}, k)=\left(\sum_{x, \text { such that } \phi(x) \in C} c_{x, \text { real }}(k)^{(+)}-c_{x, \text { real }}(k)^{(-)}\right)-c_{t p, c}(k)=0
\end{aligned}
$$

$\left|I_{i j}(k)\right|^{2} \leq{\overline{I_{i j}}}^{2}$ for all branches connecting nodes $(i, j)$

$$
v_{i, \text { real }}(k)^{2}+v_{i, \text { imag }}(k)^{2} \leq \bar{V}_{i}^{2}
$$

for all $i$ such that $i \notin N$

$$
\begin{gathered}
-K_{1 A} v_{i, \text { real }}(k)-K_{2 A} v_{i, \text { imag }}(k) \leq-\underline{V_{i}} i \in A \\
-K_{1 B} v_{i, \text { real }}(k)-K_{2 B} v_{i, \text { imag }}(k) \leq-\underline{V_{i}} i \in B \\
-K_{1 C} v_{i, \text { real }}(k)-K_{2 C} v_{i, \text { imag }}(k) \leq-\underline{V_{i}} i \in C \\
V U F_{m}(k)^{2} \leq{\overline{V U F_{m}}}^{2} \text {, for all buses } m \\
v_{i, \text { real }}(k)^{2}+v_{i, \text { imag }}(k)^{2} \leq{\overline{V_{\text {neutral }}}}^{2}, i \in N, \\
c_{x, \text { real }}(k)^{(+)^{2}}+c_{x, \text { real }}(k)^{(-)^{2}}+c_{x, \text { reactive }}(k)^{2} \leq{\overline{c_{x}}}^{2} \\
\text { for all ESS } x \\
E(x, k) \leq \overline{E_{x}} \text { for all ESS } x \\
-E(x, k) \leq-\underline{E x}_{x} \text { for all ESS } x
\end{gathered}
$$

Since the $v_{i, \text { real }}(k)^{2},\left|I_{i j}(k)\right|^{2}$ and $V U F_{m}(k)^{2}$ terms are still squared linear functions of the decision variables, we have only linear and convex constraints and a convex objective function. Hence the optimal dispatch problem can be extended to consider a multi-period time horizon while still preserving the convexity of the problem. This is especially important for computational reasons as the number of decision variables will increase significantly in a time horizon problem.

\section{APPENDIX B. EXTENSION TO DISSIMILAR CHARGING AND DISCHARGING EFFICIENCIES}

Some energy storage systems have dissimilar charging and discharging efficiencies, and for this reason separate efficiency terms for each mode were considered in [8-12]. The formulation in Appendix A is easily extended to consider this. Let $\eta_{c}(x)$ be the charging efficiency and $\eta_{d}(x)$ the discharging efficiency of the ESS $x$. Then the ESS loss term becomes:

$$
\begin{aligned}
& \sum_{k=0}^{h} \sum_{x=1}^{s}\left\{\left(1-\eta_{c}(x)\right)\left(\sqrt{c_{x, \text { real }}(k)^{(-)^{2}}+c_{x, \text { reactive }}(k)^{2}}\right)\right. \\
& \left.+\left(1-\eta_{d}(x)\right)\left(\sqrt{c_{x, \text { real }}(k)^{(+)^{2}}+c_{x, \text { reactive }}(k)^{2}}\right)\right\}
\end{aligned}
$$

This again makes use of the fact that at least one of $c_{x, \text { real }}(k)^{(+)}$ and $c_{x, \text { real }}(k)^{(-)}$will be zero at the optimal solution at each time slot $k$. The energy stored in each device is:

$$
\begin{aligned}
E(x, k)=E_{0}+\sum_{r=0}^{k} E_{g}(r) & \\
& \quad-\sum_{r=0}^{k} \frac{1}{\eta_{d}(x)} c_{x, \text { real }}(r)^{(+)} \Delta t+\sum_{r=0}^{k} \eta_{c}(x) c_{x, \text { real }}(r)^{(-)} \Delta t
\end{aligned}
$$

$E(x, k)$ is obviously a linear function of the decision variables, and the ESS loss term is still a sum of two-norms. Hence convexity is preserved. 